\title{
An alternative geometry for bolometer sensors for use at high operating temperatures
}

\author{
H. Meister ${ }^{\mathrm{a}}$, H. Langer ${ }^{\mathrm{b}}$, S. Schmitt ${ }^{\mathrm{c}}$ \\ ${ }^{a}$ Max-Planck-Institut für Plasmaphysik, Boltzmannstr. 2, D-85748 Garching, Germany \\ ${ }^{b}$ KRP-Mechatec Engineering GbR, Lichtenbergstr. 8, D-85748 Garching b. München, Germany \\ ${ }^{c}$ Fraunhofer ICT-IMM, Carl-Zeiss-Str. 18-20, D-55129 Mainz, Germany
}

\begin{abstract}
Bolometer sensors are a key component to determine the total radiation and the radiation profile in fusion devices. For future devices like ITER the need arose to develop new sensors in order to adapt to loads, in particular neutron irradiation and enhanced thermal loads. The method proposed here to deal effectively with the stresses in the absorber and its supporting membrane is to support the absorber by flexure hinges, thus allowing deformations in all dimensions and reducing stresses. First, a design for the flexure hinges is proposed. Then finite-element analyses (FEA) have been carried out to investigate expected deformations due to residual stresses from the manufacturing process as well as due to additional thermal loads at $450^{\circ} \mathrm{C}$. The results showed stress levels below the expected tensile strength of $\mathrm{Si}$. In addition, calculations show that the proposed design is expected to provide acceptable cooling time constants. Thus, prototypes based on the proposed design have been manufactured. Measurements of their deformation at room temperature are in agreement with predictions from FEA. Also, all prototypes were successfully subjected to thermal cycling up to $450^{\circ} \mathrm{C}$ without any failures, thus demonstrating a successful development. However, for future application as bolometer sensor, a change in calibration parameters is expected: a factor of five for the heat capacity and a factor of two for the cooling time constant. Further prototypes including meanders and electrical contacts need to be developed and tested to finally validate if flexure hinges are a viable means for bolometer sensors at high operating temperatures.
\end{abstract}

Keywords: bolometer, ITER, finite element analysis, sensor design

\section{Introduction}

The total plasma radiation emitted from a fusion device is most commonly measured by the bolometer diagnostic. Its sensor is based on an absorber, which is heated by the radiation emitted from the fusion plasma. The increase in temperature is monitored using a thermometer giving thus the possibility to deduce the absorbed radiational power. In case of metal resistor bolometers a thin metallic meander on the back side of the absorber provides the measurement of the temperature changes [1]. It is combined with a similar meander (using the Wheatstone-bridge configuration) situated on a reference detector, which is shielded from the direct plasma radiation, to compensate for uncertainties in the measurement due to changes in temperature of the environment or the effect of neutron radiation. This type of bolometer sensor is being used in most current day experimental devices [2-6] and also developed further for the use in ITER [7]. However, the higher thermal loads expected for ITER as compared to the ones of current devices impose constraints which could not all be overcome up to date. In particular, repeated thermal cycling up to temperatures above $200^{\circ} \mathrm{C}$ leads in the newly developed sensors to a breaking of the $\mathrm{SiN}$ membranes supporting the $\mathrm{Pt}$ absorber. The reason identified was a fatigue cycle due to a reversal of the deflection direction of the membranes between room temperature and $450^{\circ} \mathrm{C}$ in combination with high stress concentration close to the absorber corners [8].

In order to overcome these difficulties, two possible options are available. Firstly, the chosen material combination for absorber and supporting membrane has to be improved. The choice of Pt absorbers on SiN membranes is based on their superior properties with respect to neutron irradiation. However, the initial intrinsic stresses in $\mathrm{Pt}$ on $\mathrm{SiN}$ during the manufacturing process turned out to be relatively high (500 MPa tensile stress 
in Pt, as measured on test samples). Sensitivity studies using finite-element analyses (FEA) of the membrane deflections as presented in [8] revealed that these parameters, in combination with the additional stresses due to differential thermal expansion, have a major impact on the mechanical stability. An alternative material for the absorber would be $\mathrm{Au}$, as this has an intrinsic stress level below $100 \mathrm{MPa}$ [9] and transmutationeffects are not expected to change the functionality of the absorber significantly. Secondly, a support of the absorber could be devised which does not restrict its displacement due to thermal expansion and thus leads to reduced stresses.

Both options are actively being pursued. This paper deals with the second option. It describes a proposal for a suitable design to support the absorber in Section 2. Section 3 presents the FEA performed to investigate the proposed design and Section 4 analyses the expected operational parameters for a bolometer based on such a design. Section 5 then shows the results obtained from investigating the first prototype samples. Finally, Section 6 concludes with an outlook on the next steps towards the final application of the proposed design as bolometer sensor.

\section{Flexure hinges as support of a bolometer ab- sorber}

The tensile residual stress of Pt resulting from the manufacturing process and the mismatch in coefficients of thermal expansion causes the absorber to contract and bend, as Pt is the top layer of a laminate formed by the $\mathrm{Pt}$ absorber and the $\mathrm{SiN}$ membrane substrate. If this laminate was allowed to contract and bend without restrictions, the imposed stress level in the $\mathrm{SiN}$ membrane would be significantly lower. In Fig. 1, the deformed shape of the combination of a $12.5 \mu \mathrm{m}$ Pt absorber on a $1.5 \mu \mathrm{m} \mathrm{SiN} \mathrm{substrate} \mathrm{of} \mathrm{the} \mathrm{same} \mathrm{size}(1.5 \mathrm{~mm} \times 4 \mathrm{~mm})$ is shown for the case of no external deformation restrictions, on the left at room temperature and on the right at $450{ }^{\circ} \mathrm{C}$. The maximum deformations in these cases are $\Delta u_{\mathrm{x}, \max }=8 \mu \mathrm{m}, \Delta u_{\mathrm{y}, \max }=2 \mu \mathrm{m}$ and $\Delta u_{\mathrm{z}, \max }=191 \mu \mathrm{m}$ for the displacements in $x, y$ and $z$ direction, respectively. This shows that any compliant support has to cope with in-plane deformations of less than $10 \mu \mathrm{m}$ in $x$ - and $y$-direction. The z-deformations to cope with are significantly higher but would apply only if the absorber was supported at the points showing the highest difference. If the absorber is hinged at e.g. the 4 corner points, the required $z$-compliance will be close to zero.

A potential design to cope with such deformations are flexure hinges, which are widely used for micro structured sensors, e.g. for acceleration sensors [10] or mi-
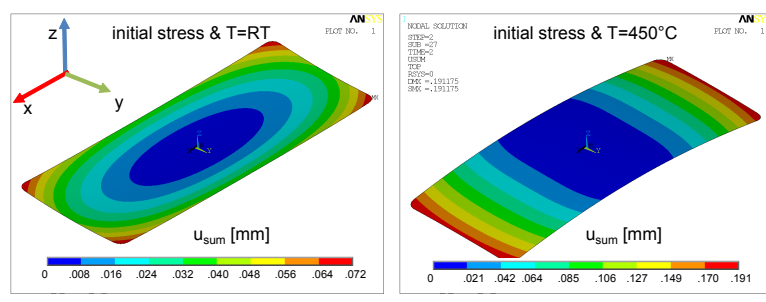

Figure 1: Free total displacement $u_{\text {sum }}$ of an absorber element consisting of a $12.5 \mu \mathrm{m}$ Pt absorber on a $1.5 \mu \mathrm{m} \mathrm{SiN} \mathrm{substrate} \mathrm{of} \mathrm{the} \mathrm{same} \mathrm{size}$ $(1.5 \mathrm{~mm} \times 4 \mathrm{~mm})$ due to intrinsic material stresses at room temperature (left) and additional thermal expansion at $450{ }^{\circ} \mathrm{C}$ (right).

cromirrors [11]. The generic design proposed is given in Fig. 2. On top, an overview of four flexure hinges

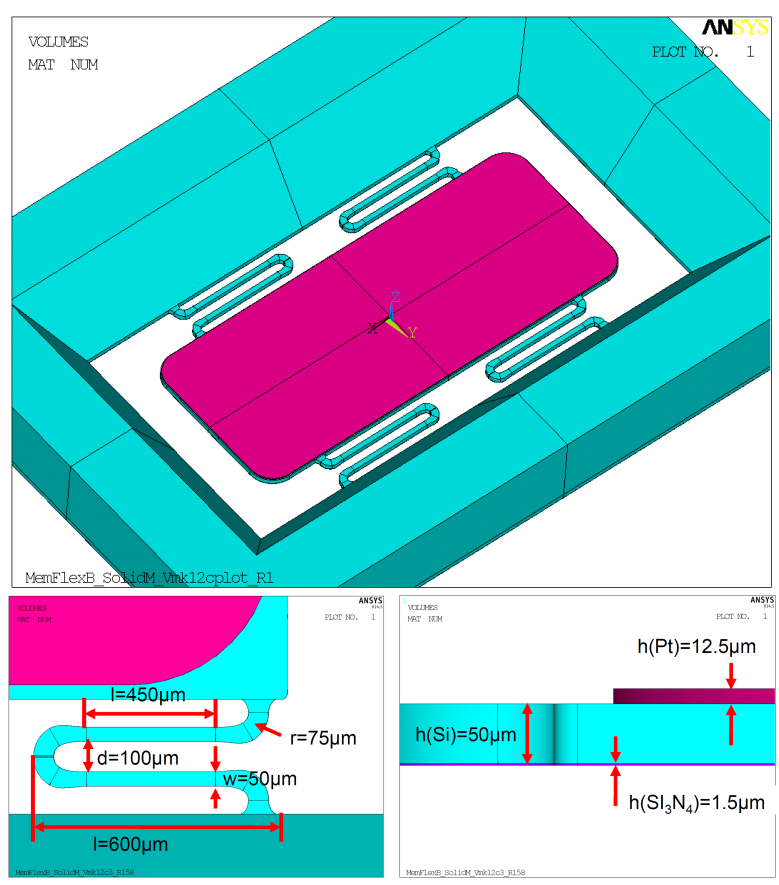

Figure 2: Overview of design to support absorber by flexure hinges (top), details on typical dimensions of flexure hinges (lower left) and thicknesses of material layers (lower right).

is shown as they attach close to the corners of the absorber. In the lower left, details on typical dimensions are given. The lower right shows the thicknesses of the various layers. From this graph it can be recognised that such a design can be realised based on the current manufacturing process [7] by stopping the etching process for the removal of the $\mathrm{Si}$ above the membrane when a remaining $50 \mu \mathrm{m}$ thickness of $\mathrm{Si}$ is achieved and applying an additional DRIE etching process to remove all material around the absorber area apart from the flexure hinges. In contrast to previous sensor prototypes the 
absorber is now placed on $50 \mu \mathrm{m} \mathrm{Si}$ and not directly on the $\mathrm{SiN}$ membrane. However, the $\mathrm{SiN}$ interlayer is still present in order to provide an isolation for the meanders which will have to be deposited on the opposite side of the Si-layer with respect to the absorber. This leaves as sole functionality for the Si-layer a mechanical one to support the absorber and therefore no detrimental effects due to neutron irradiation are expected.

The flexure hinges are designed to compensate deformations in three degrees of freedom: in-plane displacement $u_{y}$ in $y$-direction, out-of-plane displacement $u_{z}$ in $z$-direction and rotation $u_{\text {rot, } \mathrm{x}}$ around the in-plane $x$-axis (see Fig. 3, left, which uses the local hinge coordinate system). This is achieved by bending and torsion of the hinge beam (Fig. 3, right), which of course must result in stress levels below the tensile strength of $\mathrm{Si}$.

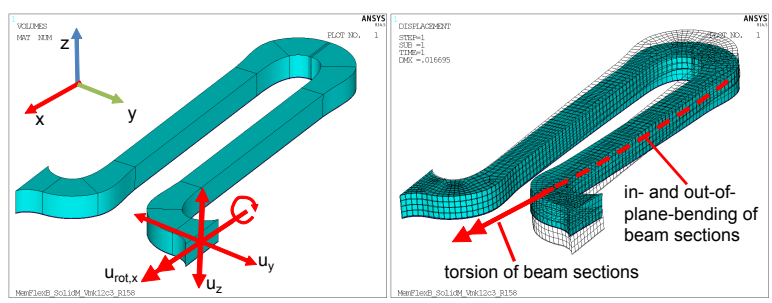

Figure 3: Principle of flexure hinge support to compensate deformations in three degrees of freedom (left) by bending and torsion of the hinge beam (right)

\section{Finite-element analyses of flexure hinge design}

The finite element analyses of this design have been performed in ANSYS. The finite-element (FE) model was realised as a solid model using ANSYS SOLID186 elements (3D 20-node solid element) and symmetry boundary conditions to be able to use only $1 / 4$ of the whole assembly for better computational performance. The model and the computational mesh is shown in Fig. 4. The loads imposed are the residual stresses from the manufacturing process realised by the "initial stress"method and for the analysis of the behaviour at high temperatures an additional thermal load of $450^{\circ} \mathrm{C}$. The analyses were performed as linear analysis. The resulting deformations due to the residual stresses and due to additional thermal loads at $450{ }^{\circ} \mathrm{C}$ are shown in Fig. 5 on the left and right, respectively. The corresponding stresses are given in Fig. 6. The highest stress levels due to residual stresses at room temperature observed are just below $500 \mathrm{MPa}$. The regions of high stress levels are located at the interface between absorber and $\mathrm{Si}$ substrate. They are also visible from the bottom of the

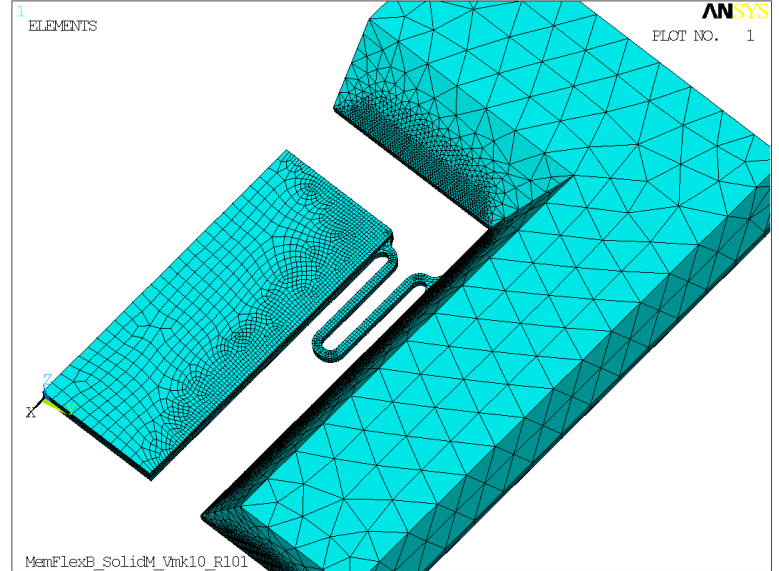

Figure 4: Model and mesh used for the analyses of the flexure hinge design.
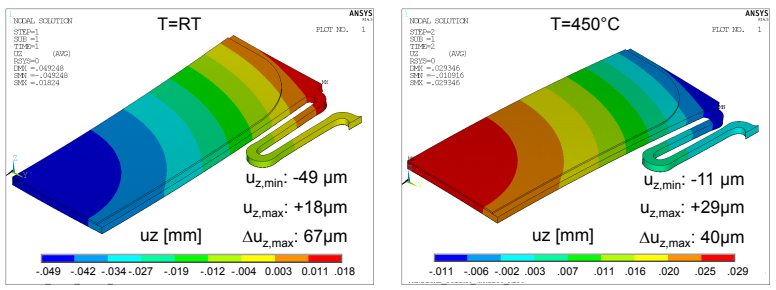

Figure 5: Deformation of flexure hinge design due to residual stresses at room temperature (left) and due to additional thermal loads at $450^{\circ} \mathrm{C}$ (right).

assembly directly below the absorber. Stresses in the hinge itself are lower. The stress levels for additional thermal loads at $450{ }^{\circ} \mathrm{C}$ decrease by about a factor of two. This is due to the mismatch of the coefficient of thermal expansion (CTE) between Pt and Si. With increasing temperature, the higher CTE of Pt results in a reduction of the initial tensile stresses in the Pt layer. The higher expansion of the $\mathrm{Pt}$ layer relative to the $\mathrm{Si}$ finally leads to a reversal of the initial bending shape. Also in this case the hinge does not show high stress values.

To judge the mechanical stability of the flexure hinge design, the calculated stress levels need to be compared to the tensile strength of Si. However, literature does not agree on a specific value for the tensile strength of Si. Depending on specimen size, test method, loading mode or defect size, respectively surface flaw size after production, values between $1 \mathrm{GPa}$ and $7 \mathrm{GPa}$ can be found. Additionally, $\mathrm{Si}$ is a brittle material for temperatures below $500{ }^{\circ} \mathrm{C}$ with a low fracture toughness of $0.7 \ldots 1.3 \mathrm{MPa} \sqrt{m}$. But even taking the lower limit into account, critical crack sizes up to $0.2 \mu \mathrm{m}$ result in a 


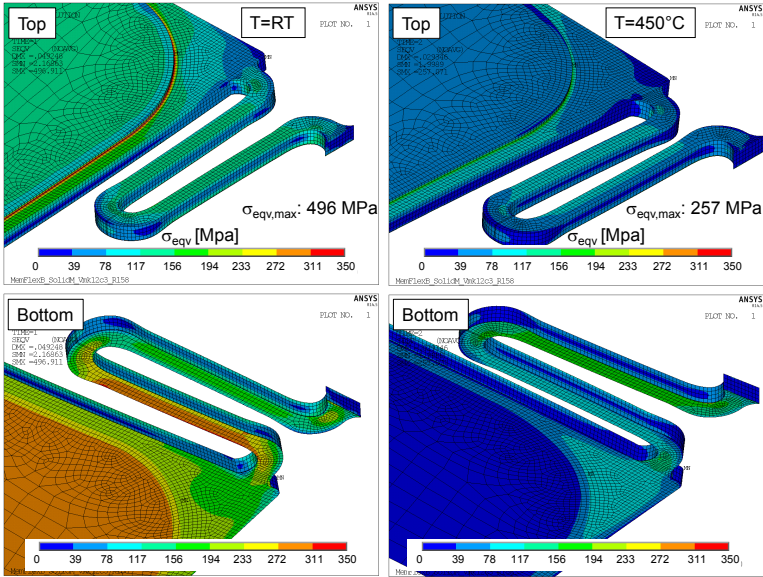

Figure 6: Stress distribution of flexure hinge design due to residual stresses at room temperature (left) and due to additional thermal loads at $450{ }^{\circ} \mathrm{C}$ (right), in both cases shown as view from top (upper) and bottom (lower).

fracture strength of $880 \mathrm{MPa}$ or higher. Thus, the flexure hinge design is expected to be mechanically stable within the analysed load cases.

\section{Expected operational parameters}

In addition to being mechanically stable, a sensitive bolometer sensor also has to feature operational parameters within acceptable ranges. The crucial parameters to check are the meander resistance, effective heat capacity and cooling time constant. These are the parameters which have to be calibrated to derive the measured radiational power [12]. Additionally, the thermal transfer time of radiated power deposited in the absorber to the meander needs to be checked as this one determines the minimum time resolution if one prefers to neglect this heat conduction during data evaluation for reasons of simplicity.

The time within which the bolometer sensor can provide a signal in response to the change of radiational power falling onto the absorber is determined by the heat transfer time from the absorber surface to the meander. The heat transfer time (or rise time) $t_{h}$ in a material with parallel bounding surfaces is given by

$$
t_{h}=\frac{d^{2}}{\kappa / c_{m} \rho}
$$

With $d$ being the thickness (in $\mathrm{m}$ ), $\kappa$ the thermal conductivity (in $\mathrm{W} \mathrm{m}^{-1} \mathrm{~K}^{-1}$ ), $c_{m}$ the specific heat capacity (in $\mathrm{J} \mathrm{kg}^{-1} \mathrm{~K}^{-1}$ ) and $\rho$ the density (in $\mathrm{kg} \mathrm{m}^{-3}$ ) of the material. Thus, $t_{h}$ of the bolometer sensor is determined by the thickness of absorber and substrate and by its material parameters. For current devices one gets $6.3 \mu \mathrm{s}$ and for the newly proposed designs $t_{h}=34 \mu \mathrm{s}$, both calculated using material parameters at room temperature. This is an increase by a factor of almost six. However, as the time resolution requirements of current and future experiments are in the order of $0.1 \mathrm{~ms}$ or longer, this difference is of no consequence. Note however, that these values were calculated for bolometer sensors based on Pt absorbers supported by $\mathrm{SiN}$ membranes or the newly proposed flexure hinges. For sensors based on Au absorbers supported by $20 \mu \mathrm{m}$ thick Mica membranes, as are still in use on some devices, typical values are $t_{h}=1.8 \mathrm{~ms}$.

As the dimensions of the absorber have not been changed, the same resistance can be achieved for the new concept as for currently operating bolometer sensors.

The additional Si supporting the absorber will add to the effective heat capacity. For a typical absorber of $1.5 \mathrm{~mm} \times 4 \mathrm{~mm}$ the $12.5 \mu \mathrm{m}$ of $\mathrm{Pt}$ give $0.21 \mathrm{~mJ} / \mathrm{K}$, the $1.5 \mu \mathrm{m}$ of $\mathrm{SiN}$ give twice $0.03 \mathrm{~mJ} / \mathrm{K}$ and the $50 \mu \mathrm{m}$ supporting $\mathrm{Si}$ give $0.49 \mathrm{~mJ} / \mathrm{K}$ when assuming typical values for density and specific heat from literature. Thus, the heat capacity will be increased by a factor of almost three.

Similarly, the change in cooling time constant will be significant. The heat transfer through the membrane or the hinges can be described using the energy balance

$$
0=\sum_{i} c_{m, i} \cdot \rho_{i} \cdot V_{i} \cdot \frac{d T}{d t}+\frac{d Q_{\mathrm{tc}}}{d t}+\frac{d Q_{\mathrm{rad}}}{d t}+\frac{d Q_{\mathrm{gas}}}{d t}
$$

where the rate of temperature change in the sensor assembly (first term on right hand side with index $i$ denoting absorber, substrate and isolation layer) is balanced by the heat transfer $Q_{\mathrm{tc}}$ through the thermal conductor, the radiational losses $Q_{\text {rad }}$ and the heat transfer through the residual gas $Q_{\text {gas }}$. Typically, radiational losses and residual gas can be neglected. $Q_{\mathrm{tc}}$ is given by

$$
\frac{d Q_{\mathrm{tc}}}{d t}=\frac{\kappa A \Delta T}{l}
$$

with the thermal conductivity coefficient $\kappa$, the cross section $A$ and length $l$ of the material, as well as the temperature difference $\Delta T$ between absorber and heat sink. The cooling time constant $\tau$ is thus given by

$$
\tau=\frac{\sum_{i} c_{m, i} \cdot \rho_{i} \cdot V_{i}}{\kappa A / l}
$$

For current devices supported by a $\mathrm{SiN}$ membrane, the mean cross section can be approximated by the mean 
outline of $13 \mathrm{~mm}$, i.e. the path on the membrane in the middle between absorber and outer heat sink, and the thickness of the membrane, yielding $1.95 \cdot 10^{-8} \mathrm{~m}^{2}$. The length is then the distance between absorber and heat sink, which is $0.5 \mathrm{~mm}$. For the hinges, the dimensions given in Fig. 2 are used to calculate a cross section of $2.5 \cdot 10^{-9} \mathrm{~m}^{2}$ and a length of $1.7 \mathrm{~mm}$. Using the heat capacities from above, the thermal conductivity of $\mathrm{SiN}$ and $\mathrm{Si}\left(15 \mathrm{~W} \mathrm{~m}^{-1} \mathrm{~K}^{-1}\right.$ and $160 \mathrm{~W} \mathrm{~m} \mathrm{~m}^{-1} \mathrm{~K}^{-1}$, respectively) and considering that there are 4 hinges supporting the absorber one gets $\tau_{\mathrm{mem}}=410 \mathrm{~ms}$ and $\tau_{\text {hinge }}=800 \mathrm{~ms}$ for membrane and hinges, respectively. This would be an increase of a factor of about 2 . In the case of a membrane supporting the absorber, measurements resulted in $\tau_{\mathrm{mem}}=450 \ldots 550 \mathrm{~ms}$ [7] and confirmed these estimates .

In principle, calibrating the sensors prior to each measurement in situ [12] offers the possibility to operate with any combination of parameters and will not be affected by the thick Si-layer as $t_{h} \ll \tau_{\text {hinge. }}$. However, in practice these calibration constants determine the sensitivity and ability to cope with measurement noise. Thus, tests on prototypes will have to demonstrate if the new support using flexure hinges is a viable means for bolometer sensors or if $\tau$ needs to be adjusted by additional heat conduction layers as is the case for current devices (not considered in the simple estimation above). Furthermore, the tests will have to show if strain-induced changes in resistance for the meander will impact the measurement. As the deformations for an absorber supported by a membrane are similar and did not show the strain-induced effects, the expectation is that this will also not be the case for this new design.

\section{Results of prototype tests}

Based on the promising FEA results, the manufacturing of prototypes with flexure hinges has been initiated. The basic design as presented in Section 2 obviously provides several parameters which can be varied and may impact on the resulting mechanical stability. These are the number of hinges, their lengths, the distance of their attachment from the corner of the absorber, the distance of the edge of the absorber from the edge of the supporting Si substrate and the corner radius of the absorber. Four different variants have been incorporated into a design for production masks to produce prototype samples. A sketch is shown in Fig. 7 of the implemented variations for four bolometer channels supported by flexure hinges, which is repeated over the surface of a 5" wafer. Based on this design, prototypes

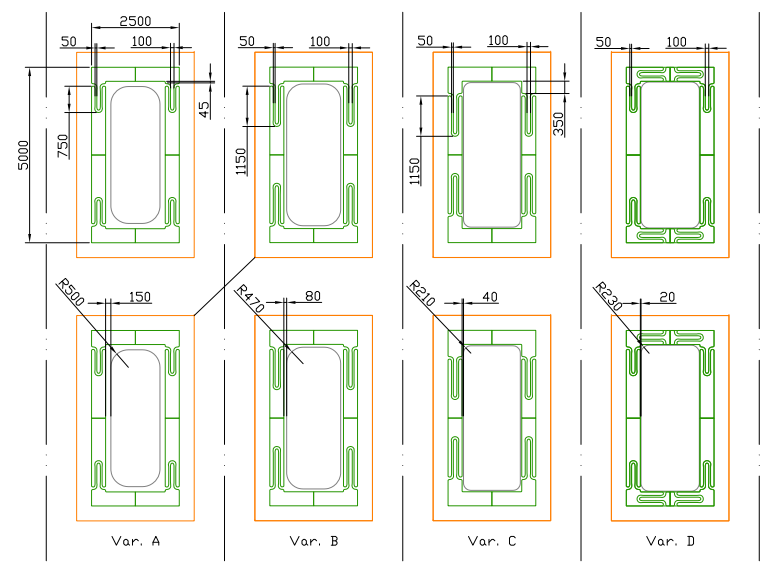

Figure 7: Design variants for absorbers supported by flexure hinges.

could be manufactured. However, for the manufacturing to be successful, an additional layer of $1.5 \mu \mathrm{m}$ of $\mathrm{Si}_{3} \mathrm{~N}_{4}$ had to be deposited between the Pt absorber and the supporting $\mathrm{Si}$ to act as diffusion barrier and improve stability. Fig. 8 shows the photograph of a whole wafer (left) and a detail of an absorber of variant A enlarged (right), demonstrating the successful process.
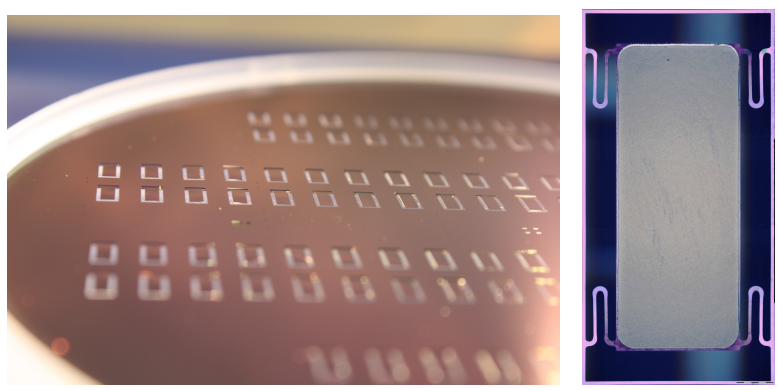

Figure 8: Photograph of a wafer of prototype absorbers supported by flexure hinges (left) and a detail showing an absorber of variant A enlarged (right).

The prototype samples produced have undergone initial tests. Firstly, white light interferometry was used to measure the out-of-plane deformation of the absorbers and flexure hinges at room temperature. Fig. 9 shows the result of the profile measurement for an absorber of variant $D$ as measured from the side of the meanders. Fig. 10 presents the evaluation of the profile measurement from Fig. 9 for a path along the length of the absorber (top) and along two hinges (middle and bottom) as well as the comparison to results from FEA for this variant. The latter has been performed assuming two different cases for the initial stress in the absorber, once for $500 \mathrm{MPa}$ and once for a slightly lower value of 


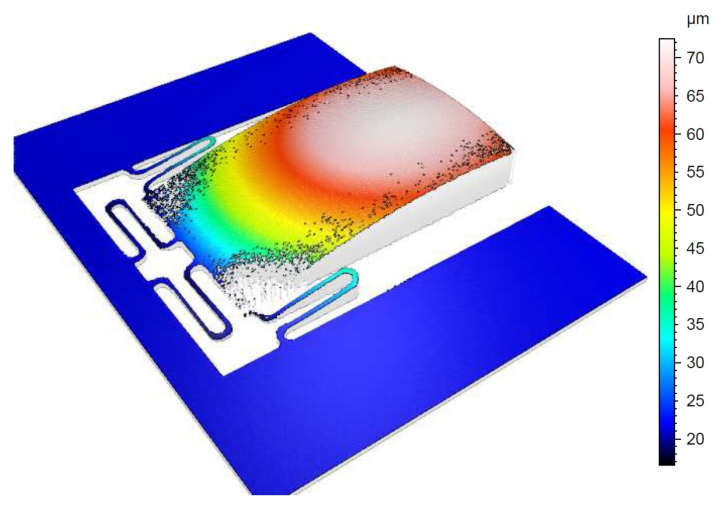

Figure 9: Result of the measurement of deformation using white light interferometry for an absorber of variant $\mathrm{D}$ as measured from the side of meanders.

$440 \mathrm{MPa}$. For both cases the results of the deformation measured compares well in shape and magnitude to the one predicted from FEA. For the case with the slightly lower value of the initial stress, the absolute magnitude of the deformation is reproduced slightly better. Considering the additional layer of $\mathrm{Si}_{3} \mathrm{~N}_{4}$, which was not taken into account for FEA and uncertainties within the material parameters, this is a very strong indication that the analysis managed to capture all relevant processes involved and that the boundary conditions and material parameters used were well chosen.

A second crucial test was the thermal cycling of the specimen. 20 thermal cycles from room temperature up to $450{ }^{\circ} \mathrm{C}$ at heating rates up to $25 \mathrm{~K} / \mathrm{min}$ and cool down to below $50{ }^{\circ} \mathrm{C}$ were applied to test their mechanical stability under thermal loads and corresponding stresses. Every specimen tolerated these loads without failure, irrespective of the geometric variations. As this is also in agreement with FEA, the development can be claimed to be a success.

\section{Conclusion}

An alternative concept has been proposed to support bolometer absorbers in order to cope with high thermal loads of future fusion devices. The concept of flexure hinges is already used for various other sensor devices and can absorb stresses due to deformations in all spatial dimensions. The proposed design has been presented and analysed using FEA. Based on the promising results, prototypes have been manufactured and tested successfully under thermal loads demonstrating not only manufacturability but also the expected mechanical stability under thermal loads up to $450{ }^{\circ} \mathrm{C}$
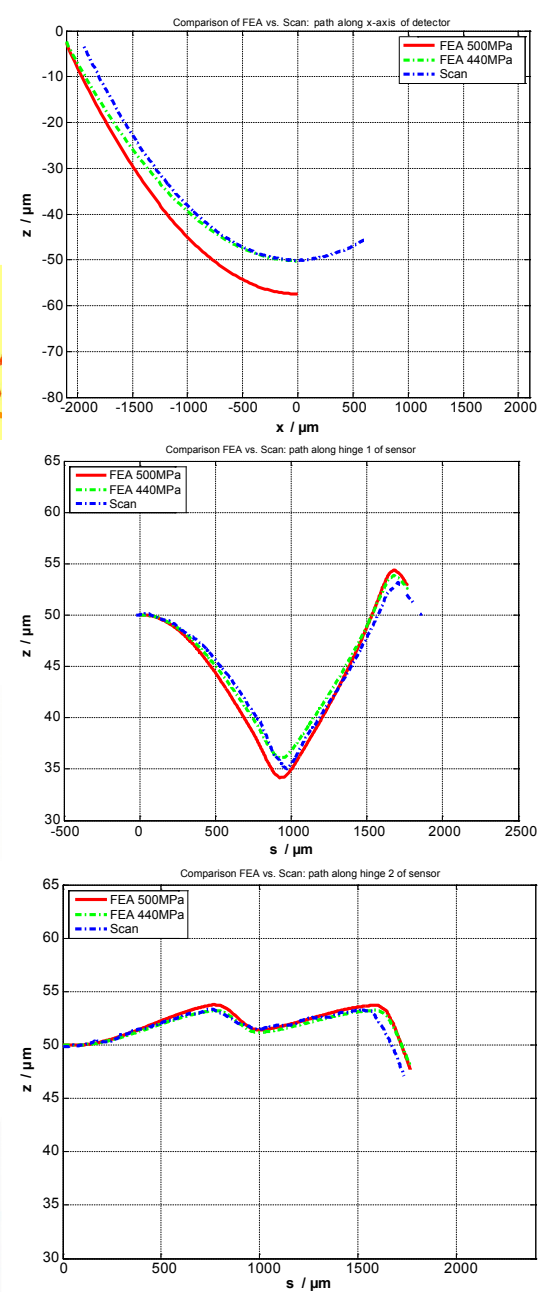

Figure 10: Comparison of the measured deformation and the prediction by FEA for the cases of $500 \mathrm{MPa}$ and $440 \mathrm{MPa}$ initial stress in the $\mathrm{Pt}$ absorber along three different paths: along the centre of the length of the absorber (top), along a hinge on the length (middle) and along a hinge on the width (bottom) of the absorber, as indicated in the pictures left of the graphs with the measurements. 
and associated stresses. However, as the demonstration of a concept for the mechanically stable support of the bolometer absorbers was the major aim so far, meanders and means to determine the bolometer signal have not been incorporated into the design, yet. The next step in the development process will be to design the layout of the meanders and the electrical signal connection using the path along the hinges to connect the meanders on the back side of the absorber and then test the resulting prototypes with respect to their parameters characterising the operation as bolometer sensor. These tests will have to show on the one hand if the estimated parameters are reproduced and on the other hand how they impact the sensitivity of a bolometer using the new sensor geometry. One particular issue to be tested experimentally on fully functional prototypes might be if shocks or vibrations during the operation of a fusion device result in oscillating noise in the bolometer signal.

\section{Acknowledgements}

This work has been funded by the German Ministry for Research and Education under Grant number 03FUS0006. The sole responsibility for the contents lies with the author.

\section{References}

[1] K. F. Mast, J. C. Vallet, C. Andelfinger, P. Betzler, H. Kraus, and G. Schramm. A low noise highly integrated bolometer array for absolute measurement of VUV and soft-X radiation. Review of Scientific Instruments, 62(3):744-750, 1991.

[2] K. McCormick, A. Huber, C. Ingesson, F. Mast, J. Fink, W. Zeidner, A. Guigon, and S. Sanders. New bolometry cameras for the JET Enhanced Performance Phase. Fusion Engineering and Design, 74:679-683, 2005.

[3] D. Zhang, R. Burhenn, R. König, L. Giannone, P. A. Grodzki, B. Klein, K. Grosser, J. Baldzuhn, K. Ewert, V. Erckman, M. Hirsch, H. P. Laqua, and J. W. Oosterbeek. Design criteria of the bolometer diagnostic for steady-state operation of the W7-X stellarator. Review of Scientific Instruments, 81:10E134, 2010.

[4] B J Peterson, A Yu Kostrioukov, N Ashikawa, Y Liu, Yuhong $\mathrm{Xu}, \mathrm{M}$ Osakabe, K Y Watanabe, T Shimozuma, S Sudo, and the LHD Experiment Group. Bolometer diagnostics for oneand two-dimensional measurements of radiated power on the large helical device. Plasma Physics and Controlled Fusion, 45(7): 1167, 2003.

[5] A. W. Leonard, W. H. Meyer, B. Geer, D. M. Behne, and D. N Hill. 2D tomography with bolometry on DIII-D. Review of Scientific Instruments, 66(2):1201-1204, 1995.

[6] Y. M. Duan, L. Q. Hu, S. T. Mao, K. Y. Chen, S. Y. Lin, and EAST Diagnostics Team. Preliminary results of the Pt foil resistive bolometer on EAST. In A. Becoulet, T. Hoang, and U. Stroth, editors, Europhysics Conference Abstracts (CDROM), Proc. of the 38 EPS Conference on Controlled Fusion and Plasma Physics, Strasbourg, 2011, volume 35G, Geneva, 2011. EPS.
[7] H. Meister, T. Eich, N. Endstrasser, L. Giannone, M. Kannamüller, A. Kling, J. Koll, T. Trautmann, P. Detemple, and S. Schmitt. Optimization of a bolometer detector for ITER based on Pt absorber on SiN membrane. Review of Scientific Instruments, 81:10E132, 2010.

[8] H. Meister, M. Kannamüller, J. Koll, A. Pathak, F. Penzel, T. Trautmann, P. Detemple, S. Schmitt, and H. Langer. Reliability issues for a bolometer detector for ITER at high operating temperatures. Review of Scientific Instruments, 83:10D724, 2012.

[9] T. C. Hodge, S. A. Bidsturp-Allen, and P. A. Kohl. Stresses in thin-film metallization. IEEE Transaction of Components, Packaging, and Manufacturing Technology - Part A, 20(2), 1997.

[10] Anton Heuberger, editor. Mikromechanik: Mikrofertigung in der Halbleitertechnologie. Springer, Berlin, 1991. Unveränderter Nachdruck der 1. Auflage, ISBN: 3540187219.

[11] B.W. Chui, T. Sun, R. Lim, D.U. S, M. Olivo, and W. Sun. Simplified monolithic process for fabricating low-cost, highfrequency, high-flatness scanning micromirrors. In: 2013 Transducers Eurosensors XXVII: The 17th International Conference on Solid-State Sensors, Actuators and Microsystems (TRANSDUCERS EUROSENSORS XXVII), pages 1036-1039, June 2013.

[12] L. Giannone, K. F. Mast, M. Schubert, NBI Team, ECRH Team, and W7-AS Team. Derivation of bolometer equations relevant to operation in fusion experiments. Review of Scientific Instruments, 73(9):3205-3214, 2002. 